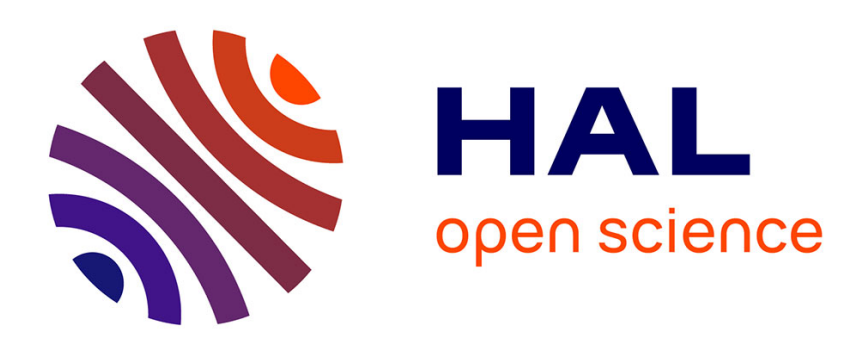

\title{
On Design of Interval Observers with Sampled Measurement
}

Denis Efimov, Emilia Fridman, Andrey Polyakov, Wilfrid Perruquetti, Jean-Pierre Richard

\section{- To cite this version:}

Denis Efimov, Emilia Fridman, Andrey Polyakov, Wilfrid Perruquetti, Jean-Pierre Richard. On Design of Interval Observers with Sampled Measurement. Systems and Control Letters, 2016. hal-01353919

\section{HAL Id: hal-01353919 \\ https://inria.hal.science/hal-01353919}

Submitted on 16 Aug 2016

HAL is a multi-disciplinary open access archive for the deposit and dissemination of scientific research documents, whether they are published or not. The documents may come from teaching and research institutions in France or abroad, or from public or private research centers.
L'archive ouverte pluridisciplinaire HAL, est destinée au dépôt et à la diffusion de documents scientifiques de niveau recherche, publiés ou non, émanant des établissements d'enseignement et de recherche français ou étrangers, des laboratoires publics ou privés. 


\title{
On Design of Interval Observers with Sampled Measurement
}

\author{
Efimov D., Fridman E., Polyakov A., Perruquetti W., Richard J.-P.
}

\begin{abstract}
New design of interval observers for continuous-time systems with discrete-time measurements is proposed. For this purpose new conditions of positivity for linear systems with sampled feedbacks are obtained. A sampled-data stabilizing control is synthesized based on provided interval estimates. Efficiency of the obtained solution is demonstrated on examples.
\end{abstract}

\section{INTRODUCTION}

State estimation in dynamical systems is a rather complicated and practically important problem [1], [2]. Sparse discrete measurement of the output for a continuous-time plant makes solution of this problem even more intricate [3], [4], [5], [6], [7]. An observer synthesis is especially problematical for the cases when the model of a nonlinear system is uncertain, and it contains parametric and/or signal uncertainties. An observer solution for these more complex situations is highly demanded in applications. Interval or set-membership estimation is a promising framework for observation in uncertain systems [8], [9], [10], [11], [12], [13], when all uncertainty is included in the corresponding intervals or polytopes, and as a result the set of admissible values (an interval) for the state is provided at each instant of time. The size of that set is related with the level of uncertainty of the plant model.

In this work the problem of design of interval sampled-data observers is studied. A peculiarity of an interval observer is that in addition to stability conditions, some restrictions on positivity of estimation error dynamics have to be imposed (in order to envelop the system solutions). In the present work we are going to use the time-delay framework for modeling and analysis of sampled-data systems [14], [15], [16], [17]. The first objective of this work is to recall the delay-dependent positivity conditions, which are based on the theory of non-oscillatory solutions for functional differential equations [18], [19], and to develop them to the time-varying sampled-data case, i.e. new sampling dependent conditions of positivity are derived. Next, continuing the research direction of [20], where the pure time-delay case has been studied, design of interval observers is given for continuous-time linear systems with discrete measurements (with time-varying sampling). The existing solutions in the field [21], [22] are based on delay-independent positivity conditions, and the interval observer constructed in [22] has a hybrid nature, which is more complicated than one proposed in the present work. Finally, following the ideas of [23] a sampled-data stabilizing control algorithm is synthesized based on interval estimates.

The paper is organized as follows. Some preliminaries are given in Section II. The delay-dependent positivity conditions for continuous systems under sampled-data measurements are presented in Section III. The interval observer design is performed for a class of linear systems (or a class of nonlinear systems in the output canonical form) with sampled measurements in Section IV. A dynamic output control design is carried out in Section V. Examples of numerical simulation are presented in Section VI.

All authors except E. Fridman are with Non-A team @ Inria, Parc Scientifique de la Haute Borne, 40 avenue Halley, 59650 Villeneuve d'Ascq, France and CRIStAL (UMR-CNRS 9189), Ecole Centrale de Lille, Cité Scientifique, 59651 Villeneuve-d'Ascq, France.

E. Fridman is with School of Electrical Engineering, Tel-Aviv University, Tel-Aviv 69978, Israel.

D. Efimov and A. Polyakov are with Department of Control Systems and Informatics, University ITMO, 49 av. Kronverkskiy, 197101 Saint Petersburg, Russia.

This work was partially supported by the Government of Russian Federation (Grant 074-U01), the Ministry of Education and Science of Russian Federation (Project 14.Z50.31.0031) and by Israel Science Foundation (grant No 1128/14). 


\section{NOTATION AND PRELIMINARIES}

In the rest of the paper, the following notation will be used:

- $\mathbb{R}$ is the Euclidean space $\left(\mathbb{R}_{+}=\{\tau \in \mathbb{R}: \tau \geq 0\}\right)$;

- $|x|$ denotes the absolute value of $x \in \mathbb{R},\|\cdot\|$ is the Euclidean norm of a vector or induced norm of a matrix;

- for a Lebesgue measurable and locally essentially bounded input $u: \mathbb{R}_{+} \rightarrow \mathbb{R}^{p}$ the symbol $\|u\|_{\left[t_{0}, t_{1}\right]}$ denotes its $L_{\infty}$ norm $\|u\|_{\left[t_{0}, t_{1}\right)}=\underset{t \in\left[t_{0}, t_{1}\right)}{e s s u p}\|u(t)\|$, or simply $\|u\|$ if $t_{0}=0$ and $t_{1}=+\infty$, the set of all such inputs with the property $\|u\|<\infty$ will be denoted as $\mathcal{L}_{\infty}^{p}$

- for a matrix $A \in \mathbb{R}^{n \times n}$ the vector of its eigenvalues is denoted as $\lambda(A)$;

- $I_{n}$ and $0_{n \times m}$ denote the identity and zero matrices of dimensions $n \times n$ and $n \times m$ respectively;

- $a \mathcal{R} b$ corresponds to an elementwise relation $\mathcal{R}$ ( $a$ and $b$ are vectors or matrices): for example $a<b$ (vectors) means $\forall i: a_{i}<b_{i}$;

- for a symmetric matrix $\Upsilon$, the relation $\Upsilon \prec 0(\Upsilon \preceq 0)$ means that the matrix is negative (semi) definite.

\section{A. Interval bounds}

Given a matrix $A \in \mathbb{R}^{m \times n}$ define $A^{+}=\max \{0, A\}, A^{-}=A^{+}-A$ and $|A|=A^{+}+A^{-}$. Let $x \in \mathbb{R}^{n}$ be a vector variable, $\underline{x} \leq x \leq \bar{x}$ for some $\underline{x}, \bar{x} \in \mathbb{R}^{n}$, and $A \in \mathbb{R}^{m \times n}$ be a constant matrix, then [24]:

$$
A^{+} \underline{x}-A^{-} \bar{x} \leq A x \leq A^{+} \bar{x}-A^{-} \underline{x} .
$$

A matrix $A \in \mathbb{R}^{n \times n}$ is called Metzler if $A_{i, j} \geq 0$ for all $1 \leq i \neq j \leq n$.

\section{B. Delay-dependent positivity}

Consider a scalar time-varying linear system with time-varying delays [18]:

$$
\begin{gathered}
\dot{x}(t)=a_{0}(t) x[g(t)]-a_{1}(t) x[h(t)]+b(t), \\
x(\theta)=0 \text { for } \theta<0, x(0) \in \mathbb{R},
\end{gathered}
$$

where $a_{0} \in \mathcal{L}_{\infty}, a_{1} \in \mathcal{L}_{\infty}, b \in \mathcal{L}_{\infty}, h(t)-t \in \mathcal{L}_{\infty}, g(t)-t \in \mathcal{L}_{\infty}$ and $h(t) \leq g(t) \leq t$ for all $t \geq 0$. For the system (2) the initial condition in (3) is, in general, not a continuous function (if $x(0) \neq 0$ ).

The following result proposes delay-independent positivity conditions.

Lemma 1. [18] (Corollary 15.7) Let $0 \leq a_{1}(t) \leq a_{0}(t)$ for all $t \geq 0$. If $x(0) \geq 0$ and $b(t) \geq 0$ for all $t \geq 0$, then the corresponding solution of (2),(3) $x(t) \geq 0$ for all $t \geq 0$.

Recall that in this case positivity is guaranteed for "discontinuous" initial conditions. The peculiarity of the condition $0 \leq a_{1}(t) \leq a_{0}(t)$ is that it may correspond to an unstable system (2). In order to overcome this issue, delay-dependent conditions can be introduced.

Lemma 2. [18] (Corollary 15.9) Let $0 \leq \frac{1}{e} a_{0}(t) \leq a_{1}(t)$ for all $t \geq 0$ and

$$
\sup _{t \in \mathbb{R}_{+}} \int_{h(t)}^{t}\left[a_{1}(\xi)-\frac{1}{e} a_{0}(\xi)\right] d \xi<\frac{1}{e},
$$

where $e=\exp (1)$. If $x(0) \geq 0$ and $b(t) \geq 0$ for all $t \geq 0$, then $x(t) \geq 0$ for all $t \geq 0$ in (2), (3).

These lemmas describe positivity conditions for a scalar system, they can also be extended to a $n$-dimensional system.

Corollary 1. [20] The system

$$
\dot{x}(t)=A_{0} x(t)-A_{1} x(t-\tau(t))+b(t), t \geq 0,
$$


where $x(t) \in \mathbb{R}^{n}, \tau: \mathbb{R}_{+} \rightarrow[-\bar{\tau}, 0]$ and $b: \mathbb{R}_{+} \rightarrow \mathbb{R}_{+}^{n}$ are Lebesgue measurable functions of time, $\bar{\tau} \in \mathbb{R}_{+}$, with initial conditions

$$
x(\theta)=0 \text { for }-\bar{\tau} \leq \theta<0, x(0) \in \mathbb{R}_{+}^{n},
$$

is positive (i.e. $x(t) \geq 0$ for all $t \geq 0$ ) if $-A_{1}$ is Metzler, $A_{0} \geq 0$, and

$$
0 \leq\left(A_{0}\right)_{i, i} \leq e\left(A_{1}\right)_{i, i}<\left(A_{0}\right)_{i, i}+\bar{\tau}^{-1}
$$

for all $1 \leq i \leq n$.

\section{Positivity OF SAMPLED SYSTEMS}

Consider a time-invariant version of (2):

$$
\begin{gathered}
a_{0}(t)=a_{0}, a_{1}(t)=a_{1}, g(t)=t, \\
h(t)=t_{k} \quad \forall t \in\left[t_{k}, t_{k+1}\right), \\
t_{k+1}=t_{k}+T_{k}, k \geq 0, t_{0}=0,
\end{gathered}
$$

where $0<T_{k} \leq \bar{T}$ is a time-varying sampling rate. Then Lemma 2 admits the following corollary.

Corollary 2. For (4), (5) let $0 \leq a_{0} \leq e a_{1}<a_{0}+\bar{T}^{-1}$. If $x(0) \geq 0$ and $b(t) \geq 0$ for all $t \geq 0$, then the corresponding solution of (2)-(5) $x(t) \geq 0$ for all $t \geq 0$.

However, as we can see from the result given below, the conditions of Corollary 2 are very conservative:

Lemma 3. Consider the system (2), (4), (5) with $x(0) \geq 0$ and $b(t) \geq 0$ for all $t \geq 0$, then $x(t) \geq 0$ for all $t \geq 0$ provided that one of the following conditions is satisfied:

(i) $a_{1} \leq 0$;

(ii) $a_{0} \geq a_{1}>0$;

(iii) $a_{1}>0, a_{1}>a_{0}, \bar{T} \leq \frac{1}{a_{0}} \ln \frac{a_{1}}{a_{1}-a_{0}}$.

Note that

$$
\lim _{a_{0} \rightarrow 0} \frac{1}{a_{0}} \ln \frac{a_{1}}{a_{1}-a_{0}}=\frac{1}{a_{1}},
$$

then condition (iii) for $a_{0}=0$ reads: $a_{1}>0$ and $\bar{T} \leq a_{1}^{-1}$.

Proof. Such a system for $t \in\left[t_{k}, t_{k+1}\right)$ for any $k \geq 0$ has solution:

$$
x(t)=e^{a_{0}\left(t-t_{k}\right)} x\left(t_{k}\right)+\int_{t_{k}}^{t} e^{a_{0}(t-s)}\left[b(s)-a_{1} x\left(t_{k}\right)\right] d s
$$

and for any $b(t) \geq 0$ the integral $\int_{t_{k}}^{t} e^{a_{0}(t-s)} b(s) d s$ is always positive, then in order to identify the conditions of positivity of the solutions the worst case $b(t)=0$ for $t \geq 0$ has to be analyzed. For this case and for $t \in\left[t_{k}, t_{k+1}\right)$, if $a_{0} \neq 0$ we obtain

$$
x(t)=\left[\left(1-\frac{a_{1}}{a_{0}}\right) e^{a_{0}\left(t-t_{k}\right)}+\frac{a_{1}}{a_{0}}\right] x\left(t_{k}\right),
$$

and for $a_{0}=0$,

$$
x(t)=\left[1-a_{1}\left(t-t_{k}\right)\right] x\left(t_{k}\right) .
$$

Therefore, for $x\left(t_{k}\right) \geq 0$ the solutions are positive if $\left(1-\frac{a_{1}}{a_{0}}\right) e^{a_{0}\left(t-t_{k}\right)}+\frac{a_{1}}{a_{0}} \geq 0$, which is true for $\left(a_{0} \neq 0\right)$

$$
a_{1} \leq 0 \text { or } a_{0} \geq a_{1}>0 \text { or } a_{1}>0, a_{1}>a_{0}, \bar{T} \leq \frac{1}{a_{0}} \ln \frac{a_{1}}{a_{1}-a_{0}},
$$

or $1-a_{1}\left(t-t_{k}\right) \geq 0$ that is satisfied for $\left(a_{0}=0\right)$

$$
a_{1} \leq 0 \text { or } a_{1}>0, \bar{T} \leq a_{1}^{-1} .
$$




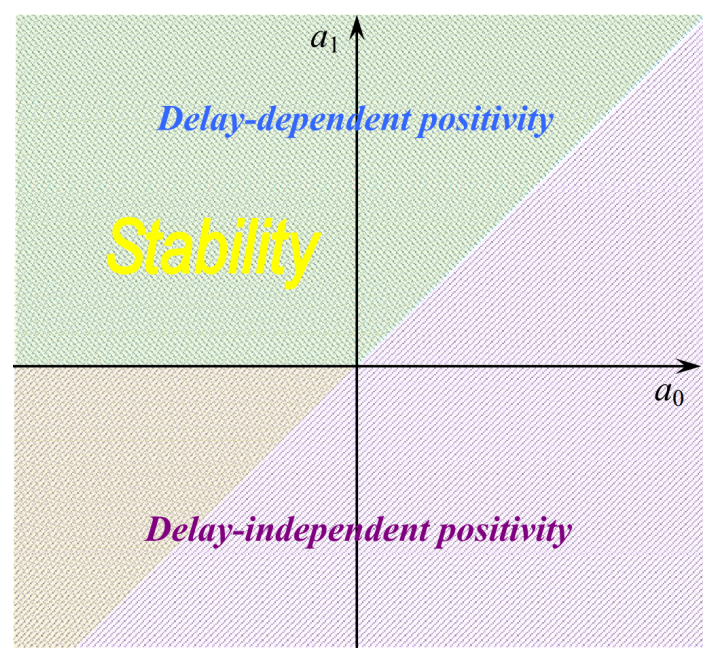

Figure 1. Illustration for lemmas 3 and 4

Using L'Hôpital's rule we derive

$$
\lim _{a_{0} \rightarrow 0} \frac{1}{a_{0}} \ln \frac{a_{1}}{a_{1}-a_{0}}=\lim _{a_{0} \rightarrow 0} \frac{\ln \frac{a_{1}}{a_{1}-a_{0}}}{a_{0}}=\lim _{a_{0} \rightarrow 0} \frac{\frac{1}{a_{1}-a_{0}}}{1}=\frac{1}{a_{1}},
$$

then the stated delay-dependent positivity conditions follow (the case for $a_{0} \neq 0$ includes $a_{0}=0$ ).

Note that the result of Lemma 3 deals only with positivity of the solutions, but not with stability, and the case of Lemma 1 is completely covered. Lemma 2 deals (implicitly through non oscillatory solution behavior) with stable positive systems, then the following extension of Lemma 3 can be proposed.

Lemma 4. Consider the system (2), (4), (5) with $x(0) \geq 0$ and $b(t) \geq 0$ for all $t \geq 0, b \in \mathcal{L}_{\infty}^{n}$, then $x(t) \geq 0$ for all $t \geq 0$ and $x(t) \in \mathcal{L}_{\infty}^{n}$ provided that one of the following conditions is satisfied:

(i) $a_{0}<a_{1} \leq 0$;

(ii) $a_{1}>0, a_{1}>a_{0}, \bar{T} \leq \frac{1}{a_{0}} \ln \frac{a_{1}}{a_{1}-a_{0}}$.

Proof. From the proof of Lemma 3, the system is stable and positive provided that $0 \leq\left(1-\frac{a_{1}}{a_{0}}\right) e^{a_{0}\left(t-t_{k}\right)}+\frac{a_{1}}{a_{0}}<1$ for $a_{0} \neq 0$ and $0 \leq 1-a_{1}\left(t-t_{k}\right)<1$ for $a_{0}=0$ (for $t>t_{k}$ ). The conditions of positivity for these coefficients are given in Lemma 3 , the conditions that they are less than 1 are as follows:

$$
a_{1}>a_{0} \neq 0 \text { or } a_{1}>a_{0}=0, \bar{T} \leq a_{1}^{-1} \text {. }
$$

Combination of these conditions gives the result.

An illustration for conditions of lemmas 3 and 4 is presented in Fig. 1.

Note that the case (i) of Lemma 4 corresponds to "delay-independent" positivity and stability conditions, which have been already investigated in the literature [21], [22]. Thus, in the present work we will be more interested in the case (ii) of Lemma 4 , then the following corollary can be formulated for a $n$-dimensional linear system:

Corollary 3. The system

$$
\begin{gathered}
\dot{x}(t)=A_{0} x(t)-A_{1} x\left(t_{k}\right)+b(t), t \in\left[t_{k}, t_{k+1}\right), \\
t_{k+1}=t_{k}+T_{k}, k \geq 0, t_{0}=0 ; A_{0}, A_{1} \in \mathbb{R}^{n \times n},
\end{gathered}
$$

where $x(t) \in \mathbb{R}^{n}, 0<T_{k} \leq \bar{T}$ is a time-varying sampling rate and $b: \mathbb{R}_{+} \rightarrow \mathbb{R}_{+}^{n}$ is a Lebesgue measurable function, for an 
initial condition $x(0) \in \mathbb{R}_{+}^{n}$ admits $x(t) \geq 0$ for all $t \geq 0$ if $-A_{1}$ is Metzler, $A_{0} \geq 0$, and

$$
0<\left(A_{1}\right)_{i, i},\left(A_{0}\right)_{i, i}<\left(A_{1}\right)_{i, i}<\left(A_{0}\right)_{i, i} \frac{e^{\left(A_{0}\right)_{i, i} \bar{T}}}{e^{\left(A_{0}\right)_{i, i} \bar{T}}-1}
$$

for all $1 \leq i \leq n$.

Stability conditions for this $n$-dimensional case can be found in [17].

Let us show how these conditions can be used for design of interval observers.

\section{INTERVAL OBSERVER DESIGN UNDER SAMPLED MEASUREMENTS}

In this section a statement of the problem is given. Next, an interval observer design is presented. And, finally, a control algorithm is synthesized based on interval estimates.

\section{A. Problem statement}

Consider a linear system with sampled measurements:

$$
\begin{aligned}
\dot{x}(t) & =A x(t)+B u(t)+d(t), \\
y(t) & =C x[h(t)]+v[h(t)],
\end{aligned}
$$

where $x(t) \in \mathbb{R}^{n}, h(t)$ is defined in $(5) ; y(t) \in \mathbb{R}^{p}$ is the system output available for sampled measurements with the noise $v \in \mathcal{L}_{\infty}^{p} ; u \in \mathbb{R}^{m}$ is the control, $u \in \mathcal{L}_{\infty}^{m} ; d \in \mathcal{L}_{\infty}^{n}$ is the system disturbing input; the constant matrices $A, B$ and $C$ have appropriate dimensions. It is assumed that for given $u$ and $d$ the system has a unique solution defined at least locally.

Assumption 1. For given inputs $u \in \mathcal{L}_{\infty}^{m}, d \in \mathcal{L}_{\infty}^{n}$ and initial condition $x(0) \in \mathbb{R}^{n}$, the corresponding solution of (6) $x(t)$ is bounded. In addition, there exist known vectors $\underline{x}_{0}, \bar{x}_{0} \in \mathbb{R}^{n}$ such that $\underline{x}_{0} \leq x(0) \leq \bar{x}_{0}$.

Boundedness of the state $x(t)$ is a usual assumption in the estimation theory [1], [2]. The assumption about a known set $\left[\underline{x}_{0}, \bar{x}_{0}\right]$ for the initial conditions $x(0)$ is standard for the interval or set-membership estimation theory [25], [8], [9], [10], [11]. We will assume that the values of matrices $A, B$ and $C$ are known, for the sampling $h(t)$ the bound $\bar{T}$ is given, the instant values of the signals $d(t)$ and $v(t)$ are unavailable. In the last subsection this assumption will be relaxed and a control will be designed ensuring boundedness of the state.

Assumption 2. There exist known signals $\underline{d}, \bar{d} \in \mathcal{L}_{\infty}^{n}$ and $\underline{v}, \bar{v} \in \mathcal{L}_{\infty}^{p}$ such that $\underline{d}(t) \leq d(t) \leq \bar{d}(t)$ and $\underline{v}(t) \leq v(t) \leq \bar{v}(t)$ for all $t \geq 0$.

Therefore, the uncertain inputs $d(t)$ and $v(t)$ in (6) belong to known intervals $[\underline{d}(t), \bar{d}(t)]$ and $[\underline{v}(t), \bar{v}(t)]$, respectively, for all $t \geq 0$.

It is required to design an interval observer,

$$
\begin{aligned}
\dot{\xi}(t)= & F[\xi(t), \underline{d}(t), \bar{d}(t), \underline{v}(t), \bar{v}(t), y(t)], \xi(0) \in \mathbb{R}^{s}, \\
& \underline{x}(t)=\underline{G}[\xi(t), \underline{d}(t), \bar{d}(t), \underline{v}(t), \bar{v}(t), y(t)], \\
& \bar{x}(t)=\bar{G}[\xi(t), \underline{d}(t), \bar{d}(t), \underline{v}(t), \bar{v}(t), y(t)],
\end{aligned}
$$

where $F: \mathbb{R}^{s+2 n+3 p} \rightarrow \mathbb{R}^{s}, \underline{G}: \mathbb{R}^{s+2 n+3 p} \rightarrow \mathbb{R}^{n}$ and $\bar{G}: \mathbb{R}^{s+2 n+3 p} \rightarrow \mathbb{R}^{n}$ are nonlinear maps ensuring existence of solutions, $s>0$, such that $\underline{x}(t) \leq x(t) \leq \bar{x}(t)$ for all $t \geq 0$ and $\underline{x}, \bar{x} \in \mathcal{L}_{\infty}^{n}$. A similar problem has been studied in [21], [22] applying a continuous-discrete observer. In the present work, a continuous sampled-data observer is constructed and the time-delay approach is used to sampled-data control design. 


\section{B. Motivating example}

Consider a motivating example introduced in [21], where the above problem has been posed for a scalar system

$$
\begin{gathered}
\dot{x}(t)=x(t)+u(t), \\
y(t)=x[h(t)]
\end{gathered}
$$

with $h(t)$ given in (5). This system is unstable for $u(t)=0$. It has been proven in [21] that this system has no interval observer of the form

$$
\begin{aligned}
\dot{\bar{x}}(t) & =-\bar{x}(t)+u(t)+2 y(t), \\
\underline{\dot{x}}(t) & =-\underline{x}(t)+u(t)+2 y(t)
\end{aligned}
$$

(more precisely, the case $u(t)=0$ has been studied in [21]).

Applying the result of Lemma 4, the following interval observer can be proposed for (7) of a form similar to (8).

Claim 1. For the system (7) with any initial condition $x(0) \in\left[\underline{x}_{0}, \bar{x}_{0}\right]$, the system

$$
\begin{gathered}
\underline{\dot{x}}(t)=\underline{x}(t)+u(t)+a\{y(t)-\underline{x}[h(t)]\}, \\
\dot{\bar{x}}(t)=\bar{x}(t)+u(t)+a\{y(t)-\bar{x}[h(t)]\}, \\
\underline{x}(0)=\underline{x}_{0}, \bar{x}(0)=\bar{x}_{0}
\end{gathered}
$$

is an interval observer, i.e. $\underline{x}(t) \leq x(t) \leq \bar{x}(t)$ for all $t \geq 0$, provided that $1<a<\frac{e^{\bar{T}}}{e^{\bar{T}}-1}$.

Proof. Introducing the interval estimation errors $\underline{e}=x-\underline{x}$ and $\bar{e}=\bar{x}-x$, we obtain

$$
\begin{aligned}
& \dot{\dot{e}}(t)=\underline{e}(t)-a \underline{e}[h(t)], \\
& \dot{\bar{e}}(t)=\bar{e}(t)-a \bar{e}[h(t)] .
\end{aligned}
$$

Next, it is straightforward to check that all conditions of Lemma 4 are satisfied for the equations describing the error dynamics.

Therefore, for any period of sampling $\bar{T}>0$ there exists $a>1$ such that the conditions of Claim 1 are satisfied and interval estimation is possible. Restricting value of the maximal sampling $\bar{T}$ it is possible to ensure boundedness and asymptotic convergence of errors $\underline{e}$ and $\bar{e}$ [15], [16]. The results of simulation for this observer are given in Section 6 .

Let us extend this idea of interval observer design to a more generic system (6).

\section{Interval estimation with sampled measurements}

The equation (6) can be rewritten as follows:

$$
\dot{x}(t)=A x(t)+B u(t)-L C x[h(t)]+L y(t)+d(t)-L v[h(t)],
$$

where $L \in \mathbb{R}^{n \times p}$ is an observer gain to be designed.

Any square matrix $Q$ can be presented as $Q=Q^{\dagger}+Q^{2}$, where $Q^{\dagger}$ is a diagonal matrix composed by all elements on the main diagonal of $Q$, and $Q^{2}$ is formed by the rest elements of $Q$ out of the main diagonal.

Assumption 3. There exists an invertible matrix $S \in \mathbb{R}^{n \times n}$ such that

$$
\begin{gathered}
-S L C S^{-1}=Q_{1}=\left[\begin{array}{cc}
\bar{Q}_{1} & 0_{l \times n-l} \\
0_{n-l \times l} & 0_{n-l \times n-l}
\end{array}\right], S A S^{-1}=Q_{0}=\left[\begin{array}{cc}
Q_{0,1} & Q_{0,2} \\
Q_{0,3} & Q_{0,4}
\end{array}\right], \\
Q_{0,1} \in \mathbb{R}^{l \times l}, Q_{0,2} \in \mathbb{R}^{l \times n-l}, Q_{0,3} \in \mathbb{R}^{n-l \times l} \\
Q_{0,4} \in \mathbb{R}^{n-l \times n-l}, \bar{Q}_{1}=\bar{Q}_{1}^{\dagger}+\bar{Q}_{1}^{2}, Q_{0,4}=Q_{0,4}^{\dagger}+Q_{0,4}^{2}
\end{gathered}
$$


where $\bar{Q}_{1}^{\dagger}=\operatorname{diag}\left[-q_{1,1}, \ldots,-q_{1, l}\right]$ with $q_{1, k}>0$ for all $k=1, \ldots, l, \bar{Q}_{1}^{2} \geq 0$, and $0<l \leq n$.

The matrices $S$ and $L$ can be found as a solution of Sylvester equation [13]. In the new coordinates $z=S x=\left[z_{1}^{\mathrm{T}} z_{2}^{\mathrm{T}}\right]^{\mathrm{T}}$, $z_{1} \in \mathbb{R}^{l}, z_{2} \in \mathbb{R}^{n-l}$ the system (6) takes the form:

$$
\begin{aligned}
\dot{z}_{1}(t)= & \bar{Q}_{0} z(t)+\bar{Q}_{1} z_{1}[h(t)] \\
& +\Sigma_{1} u(t)+\Lambda_{1} y(t)+\delta_{1}(t), \\
\dot{z}_{2}(t)= & Q_{0,3} z_{1}(t)+Q_{0,4} z_{2}(t) \\
& +\Sigma_{2} u(t)+\Lambda_{2} y(t)+\delta_{2}(t),
\end{aligned}
$$

where $\bar{Q}_{0}=\left[\begin{array}{ll}Q_{0,1} & Q_{0,2}\end{array}\right], S B=\left[\begin{array}{ll}\Sigma_{1}^{\mathrm{T}} & \Sigma_{2}^{\mathrm{T}}\end{array}\right]^{\mathrm{T}}$ and $S L=\left[\begin{array}{ll}\Lambda_{1}^{\mathrm{T}} & \Lambda_{2}^{\mathrm{T}}\end{array}\right]^{\mathrm{T}}$ are the matrices of appropriate dimensions; and the input $\delta(t)=\left[\delta_{1}^{\mathrm{T}}(t) \delta_{2}^{\mathrm{T}}(t)\right]^{\mathrm{T}}=S\{d(t)-L v[h(t)]\}$ with the initial condition $z(0)=\left[z_{1}(0)^{\mathrm{T}} z_{2}(0)^{\mathrm{T}}\right]^{\mathrm{T}}=S x(0)$ have interval bounds

$$
\begin{gathered}
\underline{z}_{0} \leq z(0) \leq \bar{z}_{0} \\
\underline{\delta}(t) \leq \delta(t) \leq \bar{\delta}(t) \quad \forall t \geq 0,
\end{gathered}
$$

where

$$
\begin{gathered}
\underline{z}_{0}=S^{+} \underline{x}_{0}-S^{-} \bar{x}_{0}, \bar{z}_{0}=S^{+} \bar{x}_{0}-S^{-} \underline{x}_{0}, \\
\underline{\delta}(t)=S^{+} \underline{d}(t)-S^{-} \bar{d}(t)-(S L)^{+} \bar{v}[h(t)]+(S L)^{-} \underline{v}[h(t)], \\
\bar{\delta}(t)=S^{+} \bar{d}(t)-S^{-} \underline{d}(t)-(S L)^{+} \underline{v}[h(t)]+(S L)^{-} \bar{v}[h(t)] .
\end{gathered}
$$

Then the following interval observer can be proposed for the representation (9):

$$
\begin{aligned}
\underline{\dot{z}}_{1}(t)= & \bar{Q}_{0}^{+} \underline{z}(t)-\bar{Q}_{0}^{-} \bar{z}(t)+\bar{Q}_{1} \underline{z}_{1}[h(t)] \\
& +\Sigma_{1} u(t)+\Lambda_{1} y(t)+\underline{\delta}_{1}(t), \\
\dot{\bar{z}}_{1}(t)= & \bar{Q}_{0}^{+} \bar{z}(t)-\bar{Q}_{0}^{-} \underline{z}(t)+\bar{Q}_{1} \bar{z}_{1}[h(t)] \\
& +\Sigma_{1} u(t)+\Lambda_{1} y(t)+\bar{\delta}_{1}(t), \\
\underline{\dot{z}}_{2}(t)= & Q_{0,3}^{+} \underline{z}_{1}(t)-Q_{0,3}^{-} \bar{z}_{1}(t)+Q_{0,4}^{\dagger} \underline{z}_{2}(t) \\
& +\left(Q_{0,4}^{2}\right)^{+} \underline{z}_{2}(t)-\left(Q_{0,4}^{2}\right)^{-} \bar{z}_{2}(t) \\
& +\Sigma_{2} u(t)+\Lambda_{2} y(t)+\underline{\delta}_{2}(t), \\
\dot{\bar{z}}_{2}(t)= & Q_{0,3}^{+} \bar{z}_{1}(t)-Q_{0,3}^{-} \underline{z}_{1}(t)+Q_{0,4}^{\dagger} \bar{z}_{2}(t) \\
& +\left(Q_{0,4}^{2}\right)^{+} \bar{z}_{2}(t)-\left(Q_{0,4}^{2}\right)^{-} \underline{z}_{2}(t) \\
& +\Sigma_{2} u(t)+\Lambda_{2} y(t)+\bar{\delta}_{2}(t),
\end{aligned}
$$

with initial conditions $\underline{z}(0)=\underline{z}_{0}, \bar{z}(0)=\bar{z}_{0}$ for the variables $\underline{z}(t)=\left[\underline{z}_{1}^{\mathrm{T}}(t) \underline{z}_{2}^{\mathrm{T}}(t)\right]^{\mathrm{T}}, \bar{z}(t)=\left[\bar{z}_{1}^{\mathrm{T}}(t) \bar{z}_{2}^{\mathrm{T}}(t)\right]^{\mathrm{T}}$ respectively. Finally interval estimates for the variable $x(t)$ can be obtained using

$$
\begin{aligned}
& \underline{x}(t)=\left(S^{-1}\right)^{+} \underline{z}(t)-\left(S^{-1}\right)^{-} \bar{z}(t), \\
& \bar{x}(t)=\left(S^{-1}\right)^{+} \bar{z}(t)-\left(S^{-1}\right)^{-} \underline{z}(t) .
\end{aligned}
$$

For all $k=1, \ldots, l$ denote

$$
q_{0, k}=\left(\bar{Q}_{0}^{+}\right)_{k, k}
$$

Theorem 1. Let assumptions 1, 2 and 3 be satisfied and

$$
q_{0, k} \leq q_{1, k}<q_{0, k} \frac{e^{q_{0, k} \bar{T}}}{e^{q_{0, k} \bar{T}}-1}
$$


for all $k=1, \ldots, l$. Then the interval observer (10), (11) for the system (6), (5) admits the relations

$$
\underline{x}(t) \leq x(t) \leq \bar{x}(t) \quad \forall t \geq 0 .
$$

If in addition there exist symmetric matrices $P \in \mathbb{R}^{2 n \times 2 n}$ and $U \in \mathbb{R}^{2 n \times 2 n}$, and matrices $X, X_{1}, P_{2}, P_{3}, R, Y_{1}, Y_{2} \in \mathbb{R}^{2 n \times 2 n}$ such that the LMIs

$$
\begin{aligned}
& {\left[\begin{array}{cc}
\bar{T}^{-1} P+\frac{X+X^{T}}{2} & X_{1}-X \\
* & \frac{X+X^{T}}{2}-\left[X_{1}+X_{1}^{T}\right]
\end{array}\right] \succ 0,} \\
& \Psi_{0} \prec 0, \Psi_{1} \prec 0, P \succ 0, U \succ 0 \text {, } \\
& \Psi_{0}=\left[\begin{array}{ccc}
\Omega_{11}-\frac{X+X^{T}}{2} & \Omega_{12}+\bar{T} \frac{X+X^{T}}{2} & \Omega_{13}+X-X_{1} \\
* & \Omega_{22}+\bar{T} U & \Omega_{23}-\bar{T}\left(X-X_{1}\right) \\
* & * & \Omega_{33}-\Pi
\end{array}\right], \\
& \Psi_{1}=\left[\begin{array}{cccc}
\Omega_{11}-\frac{X+X^{T}}{2} & \Omega_{12} & \Omega_{13}+X-X_{1} & \bar{T} Y_{1}^{T} \\
* & \Omega_{22} & \Omega_{23} & \bar{T} Y_{2}^{T} \\
* & * & \Omega_{33}-\Pi & \bar{T} R^{T} \\
* & * & * & -\bar{T} U
\end{array}\right] \\
& \Omega_{11}=\Phi_{0}^{T} P_{2}+P_{2}^{T} \Phi_{0}-Y_{1}-Y_{1}^{T}, \\
& \Omega_{12}=P-P_{2}^{T}+\Phi_{0}^{T} P_{3}-Y_{2}, \Omega_{13}=Y_{1}^{T}+P_{2}^{T} \Phi_{1}-R, \\
& \Omega_{22}=-P_{3}-P_{3}^{T}, \Omega_{23}=Y_{2}^{T}+P_{3}^{T} \Phi_{1} \text {, } \\
& \Omega_{33}=R+R^{T}, \Pi=\frac{X+X^{T}-2\left(X_{1}+X_{1}^{T}\right)}{2}
\end{aligned}
$$

are satisfied for

$$
\Phi_{0}=\left[\begin{array}{cccc}
Q_{01}^{+} & -Q_{01}^{-} & Q_{02}^{+} & -Q_{02}^{-} \\
-Q_{01}^{-} & Q_{01}^{+} & -Q_{02}^{-} & Q_{02}^{+} \\
Q_{0,3}^{+} & -Q_{0,3}^{-} & Q_{0,4}^{\dagger}+\left(Q_{0,4}^{2}\right)^{+} & -\left(Q_{0,4}^{2}\right)^{-} \\
-Q_{0,3}^{-} & Q_{0,3}^{+} & -\left(Q_{0,4}^{2}\right)^{-} & Q_{0,4}^{\dagger}+\left(Q_{0,4}^{2}\right)^{+}
\end{array}\right], \Phi_{1}=\left[\begin{array}{cccc}
\bar{Q}_{1} & 0_{l \times l} & 0_{l \times n-l} & 0_{l \times n-l} \\
0_{l \times l} & \bar{Q}_{1} & 0_{l \times n-l} & 0_{l \times n-l} \\
0_{n-l \times l} & 0_{n-l \times l} & 0_{n-l \times n-l} & 0_{n-l \times n-l} \\
0_{n-l \times l} & 0_{n-l \times l} & 0_{n-l \times n-l} & 0_{n-l \times n-l}
\end{array}\right],
$$

then $\underline{x}, \bar{x} \in \mathcal{L}_{\infty}^{n}$.

Proof. Introduce the interval estimation errors $\underline{e}=z-\underline{z}=\left[\underline{e}_{1}^{\mathrm{T}} \underline{e}_{2}^{\mathrm{T}}\right]^{\mathrm{T}}$ and $\bar{e}=\bar{z}-z=\left[\bar{e}_{1}^{\mathrm{T}} \bar{e}_{2}^{\mathrm{T}}\right]^{\mathrm{T}}$ for the observer (10) and (9):

$$
\begin{aligned}
\underline{\dot{e}}_{1}(t)= & \bar{Q}_{0}^{+} \underline{e}(t)+\bar{Q}_{0}^{-} \bar{e}(t)+\bar{Q}_{1} \underline{e}_{1}[h(t)] \\
& +\delta_{1}(t)-\underline{\delta}_{1}(t), \\
\dot{\bar{e}}_{1}(t)= & \bar{Q}_{0}^{+} \bar{e}(t)+\bar{Q}_{0}^{-} \underline{e}(t)+\bar{Q}_{1} \bar{e}_{1}[h(t)] \\
& +\bar{\delta}_{1}(t)-\delta_{1}(t), \\
\underline{\dot{e}}_{2}(t)= & Q_{0,3}^{+} \underline{e}_{1}(t)+Q_{0,3}^{-} \bar{e}_{1}(t) \\
& +\left[Q_{0,4}^{\dagger}+\left(Q_{0,4}^{2}\right)^{+}\right] \underline{e}_{2}(t) \\
& +\left(Q_{0,4}^{2}\right)^{-} \bar{e}_{2}(t)+\delta_{2}(t)-\underline{\delta}_{2}(t), \\
\dot{\bar{e}}_{2}(t)= & Q_{0,3}^{+} \bar{e}_{1}(t)+Q_{0,3}^{-} \underline{e}_{1}(t) \\
& +\left[Q_{0,4}^{\dagger}+\left(Q_{0,4}^{2}\right)^{+}\right] \bar{e}_{2}(t) \\
& +\left(Q_{0,4}^{2}\right)^{-} \underline{e}_{2}(t)+\bar{\delta}_{2}(t)-\delta_{2}(t) .
\end{aligned}
$$

It is easy to see that positivity for the variables $\underline{e}_{1}(t)$ and $\bar{e}_{1}(t)$ is guaranteed by Corollary 3 , while for the variables $\underline{e}_{2}(t)$ and $\bar{e}_{2}(t)$ the positivity follows the fact that the matrix $Q_{0,4}^{\dagger}+\left(Q_{0,4}^{2}\right)^{+}$is Metzler by construction and the rest terms in the right-hand side of $\underline{\dot{e}}_{2}, \dot{\bar{e}}_{2}$ are nonnegative provided that $\underline{e}(t) \geq 0$ and $\bar{e}(t) \geq 0$. By induction, if $\underline{e}(0) \geq 0$ and $\bar{e}(0) \geq 0$, then the relations $\underline{e}(t) \geq 0, \bar{e}(t) \geq 0$ are preserved for all $t \geq 0$ [26]. Therefore, from (11) the inclusion (12) is valid. 
In order to prove boundedness of $\underline{x}, \bar{x}$ consider the system

$$
\begin{gathered}
\dot{\zeta}(t)=\Phi_{0} \zeta(t)+\Phi_{1} \zeta[h(t)]+\Lambda y(t) \\
+\delta(t)+\Sigma u(t), \\
\Lambda=\left[\begin{array}{llll}
\Lambda_{1}^{\mathrm{T}} & \Lambda_{1}^{\mathrm{T}} & \Lambda_{2}^{\mathrm{T}} & \Lambda_{2}^{\mathrm{T}}
\end{array}\right]^{\mathrm{T}}, \\
\delta=\left[\begin{array}{llll}
\underline{\delta}_{1}^{\mathrm{T}} & \bar{\delta}_{1}^{\mathrm{T}} & \underline{\delta}_{2}^{\mathrm{T}} & \bar{\delta}_{2}^{\mathrm{T}}
\end{array}\right]^{\mathrm{T}}, \\
\Sigma=\left[\begin{array}{llll}
\Sigma_{1}^{\mathrm{T}} & \Sigma_{1}^{\mathrm{T}} & \Sigma_{2}^{\mathrm{T}} & \Sigma_{2}^{\mathrm{T}}
\end{array}\right]^{\mathrm{T}},
\end{gathered}
$$

where $\zeta=\left[\begin{array}{cccc}z_{1}^{T} & \bar{z}_{1}^{T} & \underline{z}_{2}^{T} & \bar{z}_{2}^{T}\end{array}\right]^{T}$ and the matrices $\Phi_{0}$ and $\Phi_{1}$ are defined in the theorem formulation. The introduced LMIs imply stability of this system [15], and boundedness of solutions for any bounded inputs.

Contrarily (10), (11), the interval observer proposed in [22] has hybrid dynamics and it is more complicated.

Remark 1. In order to evaluate the interval estimation accuracy, the following variable can be introduced:

$$
\Delta=\bar{z}-\underline{z}
$$

which characterizes the interval width obtained by the observer, and its dynamics is governed by differential equation:

$$
\dot{\Delta}(t)=Q_{0} \Delta(t)+Q_{1} \Delta_{1}[h(t)]+\bar{\delta}(t)-\underline{\delta}(t) .
$$

The uncertainty interval width $\bar{\delta}(t)-\underline{\delta}(t)$ serves as the external input to this system, and its stability follows the conditions of Theorem 1 .

\section{DynAmic OUtPUt-FEEDBACK STABILIZATION}

Up to now we supposed that the state $x(t)$ and the control $u(t)$ are already given and they are bounded. However, the interval estimates $\underline{x}(t), \bar{x}(t)$ can be effectively used to stabilize the uncertain system (6) [23]. Indeed, the interval observer (10), (11) guarantees interval inclusion (12) for any input $u(t)$. If a control $u(t)$ is designed such that both variables $\underline{x}(t), \bar{x}(t)$ are bounded and converge to zero, due to (12), $x(t)$ will possess the same properties. Therefore, it is possible to substitute the problem of dynamic output stabilizing control design of uncertain system (6) by the problem of stabilizing state feedback design for completely known observer (10). In this case the observer gain $L$ has to ensure positivity of the estimation errors $\bar{e}, \underline{e}$ only, and boundedness of all variables can be provided by a proper control design. Therefore, the gain $L$ has to ensure validity of Assumption 3 (solution of a Sylvester equation), while stability LMIs are provided by control selection.

Let us restrict our attention to the case of sampled control, i.e. $u(t)=u[h(t)]$ where $h(t)$ is defined in (5). According to (11), stabilization of the system in $\underline{x}(t), \bar{x}(t)$ coordinates follows its stabilization in the coordinates $\underline{z}(t), \bar{z}(t)$, then the following feedback is appropriate due to the structure of (10):

$$
u(t)=\underline{K z}[h(t)]+\bar{K} \bar{z}[h(t)]
$$

where $\underline{K} \in \mathbb{R}^{m \times n}, \bar{K} \in \mathbb{R}^{m \times n}$ are the control gains to be designed. For $\underline{K}=\left[\underline{K}_{1} \underline{K}_{2}\right]$ and $\bar{K}^{m}\left[\bar{K}_{1} \bar{K}_{2}\right]$, where $\underline{K}_{1}, \underline{K}_{2}$, $\bar{K}_{1}$ and $\bar{K}_{2}$ have appropriate dimensions, (10) can be rewritten as follows:

$$
\dot{\zeta}(t)=\Phi_{0} \zeta(t)+\Phi_{2} \zeta[h(t)]+\Lambda y(t)+\delta(t),
$$

where $\zeta=\left[\begin{array}{cccc}\underline{z}_{1}^{T} & \bar{z}_{1}^{T} & \underline{z}_{2}^{T} & \bar{z}_{2}^{T}\end{array}\right]^{T}$ and $\Phi_{0}$ are as before,

$$
\Phi_{2}=\Phi_{1}+\Sigma\left[\underline{K}_{1} \bar{K}_{1} \underline{K}_{2} \bar{K}_{2}\right]
$$


From the relations $\underline{x}(t) \leq x(t) \leq \bar{x}(t)$ we have

$$
\begin{aligned}
\left|x_{i}(t)\right| & \leq \max \left\{\left|\underline{x}_{i}(t)\right|,\left|\bar{x}_{i}(t)\right|\right\} \quad \forall 1 \leq i \leq n \\
\left|x_{i}(t)\right|^{2} & \leq\left|\underline{x}_{i}(t)\right|^{2}+\left|\bar{x}_{i}(t)\right|^{2} \quad \forall 1 \leq i \leq n \\
\|x(t)\| & \leq \sqrt{\|\underline{x}(t)\|^{2}+\|\bar{x}(t)\|^{2}} \\
& \leq \mu \sqrt{\|\underline{z}(t)\|^{2}+\|\bar{z}(t)\|^{2}} \\
& \leq \mu\|\zeta(t)\|,
\end{aligned}
$$

where $\mu=\sqrt{2\left[\left\|\left(S^{-1}\right)^{+}\right\|^{2}+\left\|\left(S^{-1}\right)^{-}\right\|^{2}\right]}$, then

$$
\|y(t)\| \leq\|C\| \mu\|\zeta[h(t)]\|+\|v[h(t)]\|,
$$

and for stabilization of $\underline{x}(t), \bar{x}(t)$ (or $\zeta(t)$ ) the signal $y(t)$ is a kind of functional perturbation, which is globally Lipschitz with respect to $\zeta[h(t)]$ (with the Lipschitz gain $\|C\| \mu$ ).

Theorem 2. Let assumptions 2 and 3 be satisfied, $\underline{x}_{0} \leq x(0) \leq \bar{x}_{0}$ for some known vectors $\underline{x}_{0}, \bar{x}_{0} \in \mathbb{R}^{n}$, and

$$
q_{0, k} \leq q_{1, k}<q_{0, k} \frac{e^{q_{0, k} \bar{T}}}{e^{q_{0, k} \bar{T}}-1}
$$

for all $k=1, \ldots, l$. Then the interval observer (10), (11) for the system (6), (5), (13) admits the relations (12). If in addition there exist symmetric matrices $P \in \mathbb{R}^{2 n \times 2 n}$ and $U \in \mathbb{R}^{2 n \times 2 n}$, and matrices $X, X_{1}, P_{2}, P_{3}, R, Y_{1}, Y_{2} \in \mathbb{R}^{2 n \times 2 n}$ such that the LMIs

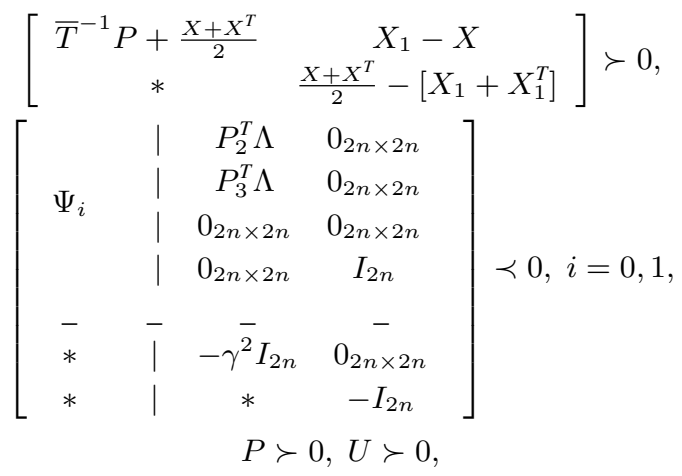

where $\Psi_{0}, \Psi_{1}$ are given in the formulation of Theorem 1 (the same for $\Omega_{11}, \Omega_{12}, \Omega_{22}, \Omega_{33}$ and $\Pi$, while $\Omega_{13}=Y_{1}^{T}+P_{2}^{T} \Phi_{2}-R$ and $\left.\Omega_{23}=Y_{2}^{T}+P_{3}^{T} \Phi_{2}\right)$, are satisfied for $\gamma<\frac{1}{\|C\| \mu}$, then $x, \underline{x}, \bar{x} \in \mathcal{L}_{\infty}^{n}$.

Proof. The proof of (12) is similar to Theorem 1.

In order to prove boundedness of $\underline{x}, \bar{x}$ note that the introduced LMIs imply internal stability of the system (14) and that the $L_{2}$ gain of the transfer $y \rightarrow \zeta$ is less than $\gamma[15]$. Since $\gamma<\frac{1}{\|C\| \mu}$ and $\|y(t)\| \leq\|C\| \mu\|\zeta[h(t)]\|+\|v[h(t)]\|$ with $v \in \mathcal{L}_{\infty}^{p}$, then by standard small-gain arguments the system (14) is asymptotically stable for $\delta=0$ and it has bounded trajectories for any bounded input $\delta$.

The main advantage of Theorem 2 with respect to Theorem 1 is that its conditions are decoupled: the observer gain $L$ ensures positivity of the estimation error dynamics, while the control gains $\underline{K}, \bar{K}$ guarantee stability.

Remark 2. Feasibility of LMIs presented in theorems 1 and 2 is discussed in [17], for instance, if the corresponding continuoustime system is exponentially stable, then the LMIs are feasible for small enough $\bar{T}$.

Remark 3. From the relation $\|x\| \leq \mu\|\zeta\|$ and the fact that under the conditions of Theorem 2 the $L_{2}$ gain of the transfer $y \rightarrow \zeta$ is less than $\gamma$, we obtain that the $L_{2}$ gain of the transfer $y \rightarrow x$ is less than $\mu \gamma<\|C\|^{-1}$. Thus, if there is an additive disturbance acting in (6) through the gain $L$, then the control (13) ensures $L_{2}$ gain with respect to that disturbance less than $\|C\|^{-1}$. Following the idea of [17] (see section 5.1), the controller gains $\underline{K}$ and $\bar{K}$ may be found from the LMIs of Theorem 2 assuming $P_{3}=\varepsilon P_{2}$, and multiplying by $\operatorname{diag}\left\{P_{2}^{-1}, \ldots, P_{2}^{-1} I_{2 n}, I_{2 n}\right\}$ from the right and its transpose from the left the second 

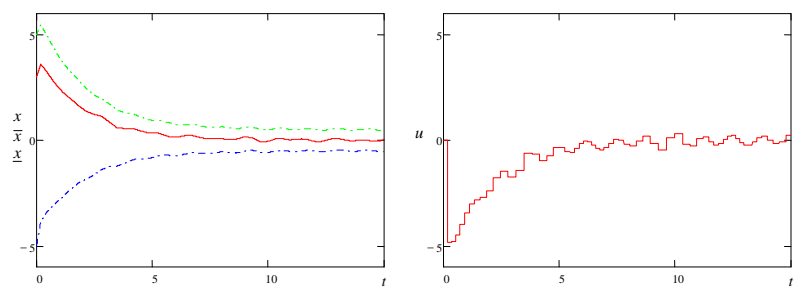

Figure 2. The results of simulation for the motivating example

LMI of Theorem 2, and by $\operatorname{diag}\left\{P_{2}^{-1}, P_{2}^{-1}\right\}$ and its transpose from the left the first LMI of the theorem.

Let us demonstrate efficiency of the developed approach for interval control and output stabilization on examples.

\section{EXAMPLES}

\section{A. Observer and control for the motivating example}

The system (7) is an example of (6) for $n=1$

$$
\dot{x}(t)=x(t)+u(t)+d(t), y(t)=x[h(t)]+v[h(t)]
$$

where $d(t) \in[-0.1,0.1]$ (for simulation $d(t)=0.1 \cos (3 t)$ ), $v(t) \in[-0.1,0.1]$ (for simulation $v(t)=0.1 \sin (5 t)$ ) and $T_{k}=\frac{\bar{T}}{2}\left(1+\sin ^{2}\left(0.5 t_{k}\right)\right)$ with $\bar{T}=0.35$. Then select:

$$
L=1.4, \underline{K}=-3, \bar{K}=-3
$$

and assume that $\left\|x_{0}\right\| \leq 5$. The interval observer (10) takes a form similar to one given in Claim 1

$$
\begin{gathered}
\dot{\dot{x}}(t)=\underline{x}(t)+u(t)+\underline{d}(t)+L\{y(t)-\underline{x}[h(t)]\}, \\
\dot{\bar{x}}(t)=\bar{x}(t)+u(t)+\bar{d}(t)+L\{y(t)-\bar{x}[h(t)]\}, \\
\underline{x}(0)=\underline{x}_{0}, \bar{x}(0)=\bar{x}_{0} .
\end{gathered}
$$

The results of simulation are shown in Fig. 2. The red solid curve represents a trajectory of the system, the blue and green dash-dot lines correspond to the interval estimates $\underline{x}(t)$ and $\bar{x}(t)$ generated by the observer.

In order to evaluate accuracy of the proposed interval observer and its relation with the maximum sampling period $\bar{T}$, introduce the variable $\delta(t)=\bar{x}(t)-\underline{x}(t)$, which represents the size of the estimated interval and characterizes precision of the interval observer. Then

$$
\dot{\delta}(t)=\delta(t)-L \delta[h(t)]+[\bar{d}(t)-\underline{d}(t)] .
$$

Using the condition (ii) of Lemma 4 we obtain for $a_{0}=1$ and $a_{1}=L$ the following restrictions on values of $L$ and $\bar{T}$ ensuring stability of $\delta$ :

$$
L>1, \bar{T} \leq \ln \frac{L}{L-1} .
$$

Increasing values of $L$ enlarges stability margins of the system and, at the same time, decreases the maximum admissible sampling period $\bar{T}$. Thus, for higher values of $L$ a better accuracy $\|\bar{x}-\underline{x}\|$ should be obtained with the price of a faster sampling. To confirm this hypothesis the results of this system simulation are shown in Fig. 3 for $\bar{d}(t)-\underline{d}(t)=1$, three different values of sampling $(\bar{T} \in\{0.231,0.347,0.555\})$ and two values of $L$ (solid lines for $L=2$ and dash lines for $L=1.5$ ). According to results presented in Fig. 3, the interval estimation accuracy is independent in $\bar{T}$ and predefined by the value of $L$ for (15). 


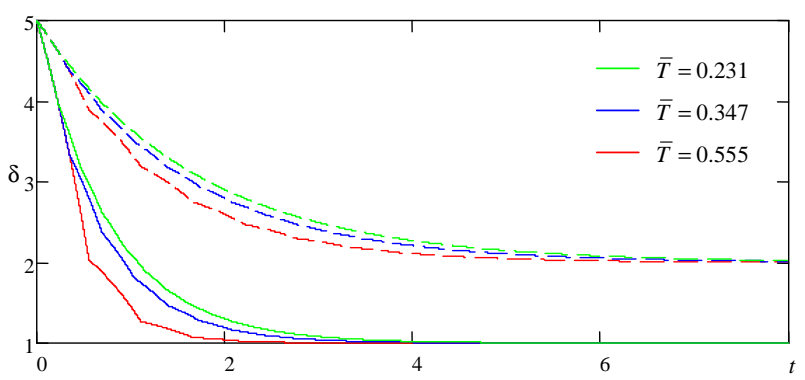

Figure 3. The results of evaluation of interval accuracy and dependence on $\bar{T}$ and $L$ for (15)
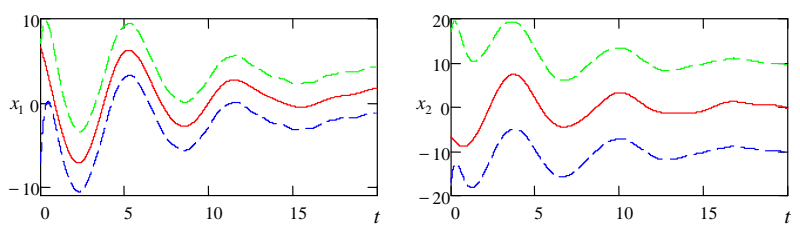

Figure 4. The results of simulation for the sampled pendulum

\section{B. A pendulum example}

Consider an example of (6) for $n=2$ from [22]

$$
\begin{gathered}
A=\left[\begin{array}{cc}
0 & 1 \\
-1 & 0
\end{array}\right], B=\left[\begin{array}{l}
0 \\
1
\end{array}\right], d(t)=\delta\left[\begin{array}{c}
\sin (t) \\
\cos (2 t)
\end{array}\right], \\
C=\left[\begin{array}{ll}
1 & 0
\end{array}\right], v(t)=V \sin (5 t), \bar{T}=0.1
\end{gathered}
$$

where $\delta>0$ and $V>0$ are the upper bounds of $d$ and $v$ available for designer. Thus, Assumption 2 is satisfied for:

$$
\begin{gathered}
\underline{d}(t)=-\left[\begin{array}{l}
\delta \\
\delta
\end{array}\right], \bar{d}(t)=-\underline{d}(t), \\
\bar{v}(t)=-\underline{v}(t)=V .
\end{gathered}
$$

Take $\left\|x_{10}\right\| \leq 10,\left\|x_{20}\right\| \leq 10$. For $L=[44]^{T}$ and

$$
S=\left[\begin{array}{cc}
\sqrt{2} & 0 \\
-1 & 1
\end{array}\right]
$$

the conditions of Assumption 3 are verified. The LMIs of Theorem 1 are satisfied for the given value of $\bar{T}$, the conditions of Theorem 2 are also satisfied for

$$
\underline{K}=-\frac{1}{8}[11], \bar{K}=-\frac{1}{16}[11]
$$

The results of simulation are shown in Fig. 4 for

$$
\delta=0.5, V=0.1, T_{k}=\bar{T}\left[0.5+0.4 \sin \left(0.5 t_{k}\right)\right]
$$

they confirm efficiency of interval estimation and validity of used delay-dependent positivity conditions.

\section{CONCLUSION}

In the paper, new positivity conditions for linear sampled systems have been proposed. These conditions are related with non-oscillatory behavior of solutions of the corresponding time-delay representation [18]. These new conditions have been employed to design interval observer for the systems with sampled measurements extending the theory of [21], [22], where a hybrid dynamics interval observer has been proposed. The results have been applied for an example from [21]. A dynamic 
output stabilizing control has been proposed based on interval state estimates. The efficacy of observers has been illustrated by numerical experiments, where dependence of the interval estimation accuracy on the maximum admissible sampling period and observer gains is also discussed.

\section{REFERENCES}

[1] G. Besançon, Ed., Nonlinear Observers and Applications, ser. Lecture Notes in Control and Information Sciences. Springer, 2007 , vol. 363.

[2] T. Fossen and H. Nijmeijer, New Directions in Nonlinear Observer Design. Springer, 1999.

[3] V. Andrieu and M. Nadri, "Observer design for Lipschitz systems with discrete-time measurements," in Proc. 49th IEEE Conference on Decision and Control, Atlanta, 2010, pp. 6522-6527.

[4] D. Deza, E. Busvelle, J. P. Gauthier, and D. Rakotopara, "High gain estimation for nonlinear systems," Systems \& Control Letters, vol. 18, no. 4, pp. 295-299, 1992.

[5] G. Goffaux, A. Vande Wouwer, and O. Bernard, "Improving continuous-discrete interval observers with application to microalgae-based bioprocess," Journal of Process Control, vol. 19, no. 7, pp. 1182-1190, 2009.

[6] H. Hammouri, N. Nadri, and R. Mota, "Constant gain observer design for continuous-discrete time uniformly observable systems," in Proc. 45th IEEE Conference on Decision and Control, San Diego, CA, 2006, pp. 5406-5411.

[7] M. Nadri, H. Hammouri, and C. Astorga, "Observer design for continuous discrete-time state affine systems up to output injection," European Journal of Control, vol. 10, no. 3, pp. 252-262, 2004.

[8] J. Gouzé, A. Rapaport, and M. Hadj-Sadok, "Interval observers for uncertain biological systems,” Ecological Modelling, vol. 133, pp. 46-56, 2000.

[9] L. Jaulin, "Nonlinear bounded-error state estimation of continuous time systems," Automatica, vol. 38, no. 2, pp. 1079-1082, 2002.

[10] M. Kieffer and E. Walter, "Guaranteed nonlinear state estimator for cooperative systems," Numerical Algorithms, vol. 37, pp. 187-198, 2004.

[11] M. Moisan, O. Bernard, and J. Gouzé, "Near optimal interval observers bundle for uncertain bio-reactors," Automatica, vol. 45, no. 1, pp. 291-295, 2009.

[12] F. Mazenc and O. Bernard, "Interval observers for linear time-invariant systems with disturbances," Automatica, vol. 47, no. 1, pp. 140-147, 2011.

[13] T. Raïssi, D. Efimov, and A. Zolghadri, "Interval state estimation for a class of nonlinear systems," IEEE Trans. Automatic Control, vol. 57, no. 1, pp. 260-265, 2012.

[14] E. Fridman, A. Seuret, and J.-P. Richard, "Robust sampled-data stabilization of linear systems: an input delay approach," Automatica, vol. 40, pp. 1441-1446, 2004

[15] E. Fridman, "A refined input delay approach to sampled-data control," Automatica, vol. 46, pp. 421-427, 2010.

[16] K. Liu and E. Fridman, “Wirtinger's inequality and Lyapunov-based sampled-data stabilization,” Automatica, vol. 48, pp. 102-108, 2012.

[17] E. Fridman, Introduction to Time-Delay Systems: Analysis and Control. Basel: Birkhäuser, 2014.

[18] R. P. Agarwal, L. Berezansky, E. Braverman, and A. Domoshnitsky, Nonoscillation theory of functional differential equations with applications. New York: Springer, 2012.

[19] A. Domoshnitsky, "Maximum principles and nonoscillation intervals for first order Volterra functional differential equations," Dynamics of Continuous, Discrete \& Impulsive Systems. A: Mathematical Analysis, vol. 15, pp. 769-814, 2008.

[20] D. Efimov, A. Polyakov, E. Fridman, W. Perruquetti, and J.-P. Richard, "Delay-dependent positivity: Application to interval observers," in Proc. ECC'15, Linz, 2015.

[21] F. Mazenc, M. Kieffer, and E. Walter, "Interval observers for continuous-time linear systems with discrete-time outputs," in Proc. 2012 American Control Conference, Montreal, 2012, pp. 1889-1894.

[22] F. Mazenc and T. Dinh, "Construction of interval observers for continuous-time systems with discrete measurements," Automatica, vol. 50, no. 10, pp. $2555-2560,2014$.

[23] D. Efimov, T. Raïssi, and A. Zolghadri, "Control of nonlinear and LPV systems: interval observer-based framework," IEEE Trans. Automatic Control, vol. 58, no. 3, pp. 773-782, 2013.

[24] D. Efimov, L. Fridman, T. Raïssi, A. Zolghadri, and R. Seydou, "Interval estimation for LPV systems applying high order sliding mode techniques," Automatica, vol. 48, pp. 2365-2371, 2012.

[25] D. Efimov, W. Perruquetti, and J.-P. Richard, "Interval estimation for uncertain systems with time-varying delays," International Journal of Control, vol. 86, no. 10, pp. 1777-1787, 2013.

[26] H. Smith, Monotone Dynamical Systems: An Introduction to the Theory of Competitive and Cooperative Systems, ser. Surveys and Monographs. Providence: AMS, 1995, vol. 41 